\title{
O uso de simuladores no auxílio do ensino-aprendizagem na enfermagem
}

\author{
Gabriela Antoneli* \\ Michele Antunes** \\ Paulo Ricardo Muniz Barros*** \\ Sandro Rigo ${ }^{* * * *}$ \\ Marie Jane Soares Carvalho* \\ Marta Rosecler Bez $z^{* * * * * *}$
}

\section{Resumo}

Este estudo objetivou verificar a perspectiva dos acadêmicos do curso de enfermagem acerca do uso de simuladores no auxílio do ensinoaprendizagem. Trata-se de um estudo transversal, exploratório, descritivo com abordagem quantitativa. A coleta de dados foi feita por meio de um questionário validado, aplicado a partir da plataforma Google Forms. A amostra foi composta por 27 acadêmicos, sendo estes de universidades públicas e privadas do Estado do Rio Grande do Sul. Os resultados demonstram que $26(96,3 \%)$ são de instituições privadas, $21(77,78 \%)$ cursam o $9^{\circ}$. semestre da graduação, $27(100 \%)$ já utilizaram a simulação,

* Discente do curso de graduação em Enfermagem da Universidade Feevale.

E-mail: gabieantonelli@gmail.com

** Doutoranda do Programa de Pós-Graduação em Informática na Educação da Universidade Federal do Rio Grande do Sul (UFRGS). Professora da Universidade Feevale. E-mail: micheleantunes@feevale.br

*** Doutorando em Computação Aplicada pela Universidade do Vale do Rio dos Sinos (UNISINOS). Professor da Universidade Feevale.

E-mail: paulobarros@feevale.br

**** Doutor em Ciência da Computação pela UFRGS. Professor da Universidade UNISINOS. E-mail: sandro.jose.rigo@gmail.com

***** Doutora em Educação pela UFRGS. Professora do Programa de Pós-Graduação em Informática na Educação da UFRGS. E-mail: mariejsc@gmail.com

****** Doutora em Informática na Educação pela UFRGS. Professora da Universidade Feevale. E-mail: martabez@feevale.br 
sendo que 26 (96,3\%) realizaram a simulação por intermédio de bonecos. A partir da análise desses dados, conclui-se que a simulação é uma metodologia ativa capaz de auxiliar no processo de ensino-aprendizagem, promovendo maior interação entre professor e acadêmico.

Palavras-chave: Enfermagem. Educação em Enfermagem. Simulação.

\section{The use of simulators to aid teaching-learning in nursing}

\section{Abstract}

The study aims to verify the perspective of nursing students about the use of simulators in the teaching-learning aid. This is a cross-sectional, exploratory, descriptive study with a quantitative approach. Data collection was performed through a validated questionnaire, applied from the Google Forms platform. The sample consisted of 27 academics, from public and private universities in the state of Rio Grande do Sul. The results show that $26(96.3 \%)$ are from private institutions, $21(77.78 \%)$ attend the 9th semester of the graduation, $27(100 \%)$ already used the simulation, and $26(96.3 \%)$ performed the simulation through dolls. From the analysis of these data, it is concluded that simulation is an active methodology capable of assisting teaching-learning, promoting greater interaction between teacher and academic, greater autonomy of the academic, stimulating psychomotor and cognitive skills of the same. The simulation presents itself as an educational resource capable of promoting a new paradigm when compared to traditional teaching.

Keywords: Nursing. Education, Nursing. Simulation.

\section{Eluso de simuladores en el auxilio de la enseñanza- aprendizaje en la enfermería}

\section{Resumen}

Este estudio objetivó verificar la perspectiva de los académicos del curso de enfermería acerca del uso de simuladores en el auxilio de la enseñanzaaprendizaje. Se trata de un estudio transversal, exploratorio, descriptivo con abordaje cuantitativo. La recolección de datos se realizó a través de un cuestionario validado, aplicado a partir de la plataforma Google Forms. La muestra fue compuesta por 27 académicos, siendo éstos de universidades públicas y privadas del Estado de Rio Grande do Sul. Los resultados demuestran que $26(96,3 \%)$ son de instituciones privadas, 21 
(77,78\%) cursan el $9^{\circ}$. semestre de la graduación, 27 (100\%) ya utilizaron la simulación, siendo que $26(96,3 \%)$ realizaron la simulación a través de muñecos. A partir del análisis de estos datos se concluye que la simulación es una metodología activa capaz de auxiliar en el proceso de enseñanzaaprendizaje, promoviendo mayor interacción entre profesor y académico.

Palabras clave: Enfermería. Educación en Enfermería. Simulación.

\section{Introdução}

O método tradicional consiste em ter o aprendiz como receptor das informações e o professor como o provedor das mesmas. Dessa forma, o foco do aprendizado está no caráter acumulativo e no professor. Esse modelo estabelece uma relação hierárquica entre o professor e o aprendiz (SCALABRINI NETO, 2017).

Algumas mudanças na educação superior, no Brasil, se deram nos anos 1990, entre elas ocorreu a reformulação curricular dos cursos de graduação, sendo esta fomentada pelas Diretrizes Curriculares Nacionais para os Cursos de Graduação. A mudança educacional de formação da enfermagem necessitava igualmente romper com a hegemonia ancorada apenas no modelo clínico (ou curativista-hospitalocêntrico), assim como direcionar o foco de formação para profissionais que assistam a saúde de modo integral (PADOVANI; CORRÊA, 2017).

Desde então, observam-se diversas iniciativas no sentido de mudar os modos de educar na área da saúde, possibilitando aos acadêmicos, assim, uma nova abordagem de ensino. Desde a utilização de métodos ativos de aprendizagem, por meio da inserção da tecnologia, até propostas mais autônomas, focadas na aprendizagem significativa.

Os métodos ativos de aprendizagem, por sua vez, supõem uma sólida formação pedagógica, psicológica e teórica, para que, por meio dessa, seja possível conhecer claramente a natureza do ato pedagógico, para, por fim, podê-lo compreender como uma prática social que demanda fundamentos científicos (ROSSO, 1992).

Alicerçado no já exposto, entende-se que, com os recursos tecnológicos disponíveis atualmente, é possível experienciar um 
aprendizado simulado, capaz de desenvolver o raciocínio clínico, e o olhar crítico para situações que, posteriormente, serão vivenciadas pelos discentes em campo de prática.

Partindo do princípio atual da relação do ensinoaprendizagem, a qualificação e formação dos profissionais da área da saúde deve disponibilizar novas possibilidades, assim como novas ferramentas para que as mesmas possam garantir melhores resultados durante a formação. A utilização da metodologia de simulação realística mostra-se como um fator importante para a melhora no desempenho, bem como, a redução de erros (FERREIRA; CARVALHO; CARVALHO, 2015).

Bez (2013) identifica ser uma boa estratégia a apresentação de conteúdos por meio da simulação de casos clínicos (fictícios ou reais), pois os professores dispõem da liberdade de criar casos que contemplem diversos temas, disponibilizando os mesmos aos alunos por intermédio da internet. Ainda, de acordo com Ziv et al. (2005) e Bradley (2006), simuladores para o ensino na área da saúde podem ser vistos como ferramentas que permitem aos docentes manter o controle total das situações e dos cenários clínicos, para que a simulação se torne fiel à realidade. Aspectos como as responsabilidades políticas, a regulamentação da profissão e as expectativas da sociedade devem ser levados em consideração.

No Brasil, a simulação realística já vem sendo utilizada em hospitais, como o Hospital Albert Einstein e o Sírio Libanês, ambos no Estado de São Paulo. Um estudo realizado pelo National Training Lab. Bethel revelou que a leitura possibilita a retenção de aprendizado de $5 \%$, a oratória $10 \%$, o áudio visual $20 \%$, a demonstração $30 \%$, a discussão em grupo $50 \%$, a prática monitorada $75 \%$ e a atuação na prática 90\%. Esses resultados mostram o impacto que o método da simulação realística pode produzir em forma de ensinoaprendizagem (FERREIRA; CARVALHO; CARVALHO, 2015).

Tendo a área da saúde como foco deste trabalho, Stanford (2010) pode ser citado. O mesmo apresenta as vantagens da 
simulação em sala de aula, sendo elas: habilidade de avaliar e refletir sobre atividades realizadas; oportunidade de experimentar situações de crises antes que as mesmas ocorram em sua prática clínica; ociosidade da criação de situações artificiais, criando, assim, situações que seriam impossíveis em cenários reais.

Para tanto, o objetivo geral deste estudo foi verificar a perspectiva dos acadêmicos do curso de Enfermagem acerca do uso de simuladores no auxílio no processo de ensino-aprendizagem.

\section{Referencial teórico}

Atualmente, constata-se que os procedimentos de ensino são tão importantes quanto os próprios conteúdos de aprendizagem. Por conseguinte, as técnicas do ensino tradicional, ou método tradicional, passam a fazer parte do alvo teórico, não só da área da educação, como da comunidade intelectual que busca identificar suas deficiências, visando propor novas metodologias de ensinoaprendizagem (PAIVA et al., 2016).

A simulação é uma tecnologia pertinente ao ensinoaprendizagem, pois envolve a replicação da realidade. Trata-se de uma metodologia ativa que engloba um processo de organização, planejamento, definição de estratégias, instrumentos e ferramentas específicas, para que o acadêmico qualifique seu aprendizado (ARAÚJO; QUILICI, 2012). Por meio da utilização da simulação, encontra-se a possibilidade de desenvolvimento do raciocínio clínico, do pensamento crítico, da problematização e da tomada de decisão em prol da segurança do paciente, com foco nas práticas de saúde. Dessa forma, a simulação pode ser integrada nos planos de ensino de modo a transversalizar os currículos da área da saúde, consolidando-se como uma tecnologia indispensável para a formação dos futuros profissionais da saúde (SANCHES, 2016).

Os simuladores são ferramentas utilizadas no processo de ensino-aprendizagem, reproduzem parcial ou totalmente a realidade. E podem ser divididos em três grupos: simuladores de pacientes (bonecos/manequins), pacientes simulados (atores), e 
programas de software (paciente virtual) (PRETO; MAGALHÃES; FERNANDES, 2010).

Ainda no contexto da simulação, existe a possibilidade do uso de pessoas (atores), os chamados pacientes simulados. Estes são utilizados para o treinamento de habilidades como: exame físico e entrevista, podendo ser realizado com pessoas que se disponham a interpretar (DUDLEY, 2012).

Em contrapartida, a simulação computacional (do tipo paciente virtual) é, de acordo com Jong e Joolingen (1998, p. 2), "um programa que contém um modelo de um sistema (natural ou artificial) ou um processo". Este suporta exemplos de prática que incluem o desenvolvimento de hipóteses, formulação de questões, revisão de teoria e coleção de dados. A simulação pode ser definida, então, como a representação ou reprodução simplificada de um processo, evento ou cenário real (BEZ, 2013).

Os autores Ziv et al. (2005) definem a técnica de simulação como um objeto de representação total ou parcial de uma tarefa replicada. Bass (2006) complementa esta definição ao referir-se aos modelos computacionais para estudo e previsão de comportamentos ou eventos, disponibilizando de uma gama ampla de aplicações, sendo estas usadas principalmente na área da educação.

Em decorrência dos avanços tecnológicos, as práticas simuladas em saúde vêm se mostrando importantes no ensinoaprendizagem. Isto fez que emergisse o interesse da indústria em construir simuladores com a capacidade de reproduzir a fisiopatologia, a fim de realizar o treinamento de futuros profissionais de saúde.

\section{Procedimentos metodológicos}

Trata-se de um estudo transversal, exploratório, descritivo, com abordagem quantitativa. A população do estudo foi composta por acadêmicos do curso de graduação de Enfermagem, de diferentes instituições públicas e privadas da região do Vale do Rio dos Sinos. A mostra foi composta por 27 acadêmicos, que 
preencheram os critérios de inclusão/exclusão. Os critérios de inclusão foram considerados: acadêmicos acima de 18 anos de idade, de ambos os sexos, matriculados no curso de Enfermagem, e que já tenham utilizado algum simulador durante o ensino. Como critérios de exclusão foram: acadêmicos menores de 18 anos de idade, que não estejam matriculados no curso de Enfermagem, e que não tenham realizado práticas com o uso de simulador.

A coleta de dados foi realizada por meio de um formulário instituído na plataforma “Google Forms". O Formulário elaborado foi divulgado em uma rede social e o indivíduo somente pôde responder o formulário uma vez. O banco de dados foi desenvolvido em uma planilha do Excel e, posteriormente, transferido para o programa estatístico Statistical Package for the Social Sciences (SPSS) versão 24.

O presente estudo respeitou as questões éticas determinadas pela resolução n . 466/2012, do Conselho Nacional de Saúde, que determina os aspectos quando uma pesquisa com seres humanos é realizada.

\section{Análise e discussão dos dados}

Neste estudo participaram acadêmicos de enfermagem, de instituições públicas e privadas do Estado do Rio Grande do Sul. A amostra foi composta por 27 (100\%) estudantes, na sua maioria, jovens, com uma média de 28,41 anos de idade, e desvio padrão de $\pm 7,05$. No que se refere à universidade, grande parte é de instituições privadas 26 (96,3\%), 21 (77,78\%) cursam o $9^{\circ}$. semestre da graduação, $21(100 \%)$ já utilizaram a simulação, sendo que 26 $(96,3 \%)$ a realizaram com bonecos.

No que tange ao ensino e ao aprendizado em Enfermagem, as instituições notaram com o passar dos anos a necessidade da utilização de métodos ativos de aprendizagem, sendo que na área da saúde o que mais se destaca é o uso da simulação. A simulação está inserida no currículo de enfermagem para o ensino de procedimentos clínicos, bem como, técnicas correspondentes 
à área de atuação. As simulações que usam bonecos acabam por facilitar a aquisição de habilidades para os cuidados dos pacientes, propiciando aos acadêmicos um ambiente seguro e interativo (GOMES; GERMANO, 2007).

No trabalho de Bez (2013), que tem por objetivo a implementação de métodos ativos de aprendizagem nas escolas de medicina, ao questionar os participantes sobre a facilidade de aprendizado no uso de simuladores, neste caso computacionais, constatou-se que $93,33 \%$ concordaram com a afirmativa, e 6,67\% discordaram desse fator. Corroborando a autora citada, o estudo de Costa (2015), em relação à satisfação dos acadêmicos com a inserção da simulação realística no ensino-aprendizagem, é de 97,30\% que se mostraram satisfeitos contra $2,70 \%$ que se mostraram indiferentes.

A respeito do fato de o aluno conseguir relacionar as práticas vivenciadas com as ensinadas por simulação, 16 (59,26\%) concordam com essa afirmativa, nove (33,33\%) discordam e dois $(7,41 \%)$ são indiferentes. De acordo com o que foi descrito no trabalho de GABA (2004), a simulação no processo de ensino e aprendizagem em saúde é um processo educativo, que substitui, no início da aprendizagem, o encontro com o paciente real. Sendo assim, o ensino ancorado em simulação contribui para o desenvolvimento das habilidades dos acadêmicos, ensinando-os a reconhecer as próprias limitações, sem colocar qualquer paciente em risco.

O trabalho de Costa (2015) corrobora a ideia dos autores citados, pois ao realizar uma pesquisa com os 48 acadêmicos e seis professores sobre a inserção da simulação no ensino, observa-se que $63,89 \%$ percebem que os cenários da simulação se aproximam muito da realidade do atendimento em saúde, 22,22\% acreditam que a simulação permite a aproximação entre a teoria e a prática, e $13,89 \%$ percebem que a simulação permite aprender com os erros.

Acredita-se, então, que o uso da simulação em sala de aula seja um método benéfico, não apenas para os acadêmicos, mas também para o professor que, por sua vez, tem a oportunidade de unir teoria e prática sem que um paciente seja exposto a possíveis danos, sejam estes físicos ou emocionais. 
Os acadêmicos compreendem as práticas de saúde de forma mais fácil por meio da simulação, segundo respostas do questionário, pois $19(70,37 \%)$ dos acadêmicos concordaram plenamente e 8 (29,63\%) concordaram com a afirmação.

Um estudo foi realizado com um simulador do tipo paciente virtual por Orton e Mulhausen (2008), em mais de 42 instituições de ensino, com $68 \%$ representando acadêmicos de medicina e $25 \%$ residentes de medicina, de faixa etária entre 20 e 29 anos, sendo $59 \%$ do sexo feminino e 29\% masculino. Este constatou que $91 \%$ concordaram que a simulação proposta atingiu o objetivo do ensino e, em relação ao conteúdo teórico e prático exposto na simulação, apenas 4\% discordaram em relação ao uso da simulação, não acreditando que a mesma consiga relacioná-los.

Já na questão sobre o ensino por simulação ter semelhança com a realidade do trabalho que será desenvolvido, 15 (55,56\%) dos acadêmicos concordaram plenamente; 10 (37,04\%) concordaram e apenas dois $(7,41 \%)$ se mostraram indiferentes à afirmação.

Segundo Costa (2015), 43,48\% dos alunos entrevistados acreditam que a simulação permite fixar os conhecimentos, bem como, possibilita a reflexão sobre a prática; 30,44\% afirmam que a simulação relaciona bem teoria e prática, que deveria ser utilizada em mais matérias na graduação e é uma metodologia dinâmica que por si só promove a integração; e por fim, 26,08\% relacionaram a simulação como metodologia que permite a aproximação da realidade, e proporciona uma vivência prévia da prática.

Quando perguntados sobre as habilidades individuais serem essenciais para as práticas de saúde, 25 (92,59\%) dos acadêmicos concordam plenamente com a afirmação, contra dois $(7,41 \%)$ que apenas concordam. O estudo de Sousa (2015), que buscou identificar a experiência de simulação clínica avançada em enfermagem, ao perguntar a 43 acadêmicos $(83,7 \%$ do sexo feminino e 16,3\% do sexo masculino), sobre o desenvolvimento de habilidades e sobre a importância das mesmas no uso da simulação, obteve como resultado: $34,9 \%$ discordaram totalmente; $7 \%$ discordaram 
da afirmação; 9,3\% se mostraram indecisos; 41,9\% concordaram e 6,9\% concordaram plenamente. Encontrou-se então um nível variável de satisfação com a utilização da simulação, em se tratando de habilidades.

Porém, ao falarmos em habilidades, não devemos esquecer que um profissional da enfermagem não deve apenas possuir habilidades práticas, mas também afetivas. Com isso, como descrito por Archanjo e Correa (2011), quando o professor utiliza o método pedagógico de espelhamento em si mesmo, por meio de simulações, acaba por favorecer reflexões atitudinais, apreendendo melhor a complexidade humana.

$\mathrm{Na}$ questão 5, sobre o fato de o acadêmico acreditar possuir um melhor rendimento utilizando a simulação ao invés do ensino tradicional, $17(62,96 \%)$ concordam plenamente, $9(33,33)$ concordam e um $(3,7)$ se mostra indiferente.

O trabalho de Ferreira et al. (2018) buscou avaliar o conhecimento, satisfação e autoconfiança dos acadêmicos dos cursos de medicina e enfermagem, por meio da simulação realística. Foram 51 participantes de ambos os cursos, sendo 28 estudantes do curso de graduação de enfermagem e 23 estudantes do curso de medicina. Obteve-se o seguinte resultado: 85,25\% dos estudantes do curso de enfermagem e $83,50 \%$ dos estudantes de medicina acreditam que a simulação estimula a autoconfiança, melhora o rendimento no aprendizado, e é benéfica como metodologia de ensino.

Já o trabalho de Pereira (2016) sobre a criação, desenvolvimento, aplicação e validação de um simulador computadorizado de realidade virtual, com 21 acadêmicos de odontologia, obteve que $100 \%$ acreditam que "o simulador trouxe mais segurança para a realização da técnica em pacientes" e "o simulador é uma metodologia eficaz de aprendizado". Logo, a simulação favorece o acesso à informação e a novos estilos de raciocínio, bem como, à construção do conhecimento. Os processos tradicionais de aprendizagem acabam por se tornar obsoletos com o surgimento de novas possibilidades de criação, levantando, 
assim, um questionamento dos modelos focados apenas na simples transmissão dos saberes (LÉVY, 2007).

$\mathrm{Na}$ questão 6, sobre desenvolver com mais facilidade o trabalho em equipe, $16(59,26 \%)$ concordam plenamente, 8 (29,63\%) concordam e três $(11,11 \%)$ se mostram indiferentes. O trabalho de Coutinho et al. (2014), buscou construir e validar uma escala de feedback associada à simulação. A população da pesquisa constou com 209 acadêmicos de enfermagem (81,3\% do sexo feminino e 18,7\% do sexo masculino). Como resultados, obteve-se que 99,5\% dos acadêmicos concordam que a simulação contribui para estruturar o pensamento clínico; 99,1\% concordam que a simulação ajuda a identificar dificuldades na assistência e 98,1\% acreditam que a simulação aumenta o potencial do trabalho em equipe.

$\mathrm{Na}$ enfermagem, indícios de discussões sobre trabalho em equipe datam de 1950, onde o intuito era diminuir a fragmentação do cuidado e os problemas de falta de pessoal (PEDUZZI; CIAMPONE, 2010). Muitos dos estudos que retratam trabalho em equipe acabam por focar algumas características, como: comunicação, interação, articulação de ações, respeito mútuo e confiança (BRANDT, et al., 2014).

A pesquisa de Versiani e Fachin (2007) corrobora a afirmação mencionada, onde o trabalho em equipe por meio da simulação foi citado como fonte de aprendizado, uma vez que ressaltou a importância de ouvir uns aos outros. Nesse sentido, a equipe se configurou como um dos principais facilitadores, pois retira o professor do papel de transmissor do conhecimento. Com base no que foi exposto, observa-se, então, que estimular o trabalho em equipe em sala de aula é uma forma de ampliar o conhecimento, por meio de trocas de ideias e pensamentos sobre um mesmo assunto.

Quando questionados a respeito de como cooperar e compartilhar o conhecimento de forma mais eficaz por meio da simulação, 14 (51,85\%) concordaram plenamente, nove $(33,33 \%)$ concordaram e quatro $(14,81 \%)$ se mostraram indiferentes.

De acordo com Belhot, Figueiredo e Malavé (2001), ao acumular conhecimento os acadêmicos devem mostrar a habilidade 
de reproduzi-lo em outras situações. A conquista do conhecimento envolve uma construção ativa, os acadêmicos são estimulados a estabelecerem um conjunto de relações por si próprios. Compartilhar o conhecimento significa melhorar os meios de aprendizagem.

O estudo de Costa (2015), ao avaliar as expectativas de 48 acadêmicos quanto à utilização da simulação no ensinoaprendizagem com o objetivo de identificar se a mesma interferia de forma positiva ou negativa no que diz respeito à dinâmica do aprendizado e do compartilhamento do conhecimento, obteve o seguinte resultado: $70,27 \%$ dos acadêmicos apresentaram grandes expectativas; $2,70 \%$ se mostraram indiferentes; $27,03 \%$ apresentaram média expectativa.

Já o estudo de Akhu-zaheya et al. (2012) buscou identificar o efeito da simulação de alta fidelidade no suporte básico de vida. A pesquisa foi realizada com 110 acadêmicos de enfermagem, separados em grupo controle (58 acadêmicos) e grupo experimental (52 acadêmicos). Quanto à retenção, aquisição e compartilhamento do conhecimento, obtiveram-se resultados satisfatórios em ambos os grupos, $53 \%$ no grupo controle que utilizou ensino tradicional e simulação e $47 \%$ no grupo experimental que usou somente a simulação. Logo, concluíram que a integração da simulação no método tradicional de ensino pode ser eficaz, pois minimiza o déficit de escolaridade, melhora a qualidade da educação e contribui para a segurança do paciente.

Ferreira, Carvalho e Carvalho (2015) salientam que o uso da simulação direciona e propicia uma ruptura do modelo tradicional de ensino. Sendo assim, o conhecimento é compartilhado entre todos os envolvidos e, com isso, são exigidas habilidades capazes de promover uma melhoria real na compreensão do conteúdo.

Acredita-se que os acadêmicos, por meio da cooperação durante a simulação, acabem por compartilhar seus conhecimentos sem mesmo terem a intenção de fazê-lo, acontece de forma natural e dinâmica. O que, por fim, facilita o aprendizado e a retenção do que se está aprendendo. 
Os acadêmicos foram confrontados sobre se sentirem seguros ao realizar práticas por meio da simulação. Como resultados, $15(55,56 \%)$ concordaram plenamente, 11 (40,74\%) concordaram e um $(3,7 \%)$ se mostrou indiferente.

No estudo de Couto (2014), sobre simulação realística no ensino de emergências pediátricas na graduação, após discussão com os 144 acadêmicos que participaram da pesquisa, pôde-se observar que $95 \%$ adquiriram mais confiança nas práticas por simulação, contra apenas $5 \%$ que se mantiveram indiferentes à prática.

O estudo de Smith e Roehrs (2009), realizado com 68 alunos de enfermagem, com o intuito de identificar a satisfação e autoconfiança a partir da simulação de alta fidelidade, constatou que $90 \%$ dos alunos se sentiram mais seguros após a simulação, e 10\% encontraram dificuldades na resolução dos problemas por simulação.

A simulação permite que os acadêmicos de enfermagem participem de experiências intensas de aprendizagem, ganhando confiança para cuidar dos pacientes e para tomar qualquer decisão (CLARO, et al., 2011). Ao realizar a simulação clínica, o acadêmico se deparará com situações semelhantes às que encontrará na vida real. E, para conseguir agir, deverá controlar seus medos e inseguranças (TERRA, et al., 2006). Como visto, nota-se que a prática na simulação auxilia o acadêmico não só na retenção do conhecimento, mas também no ganho de confiança, para que possa enfrentar o mundo enquanto profissional.

Quando perguntados se os questionados acreditam que o uso da simulação no ensino reflete mais segurança ao paciente nas práticas de saúde, 19 (70,37\%) concordaram plenamente, sete $(25,93 \%)$ concordaram, e um $(3,7 \%)$ é indiferente.

O trabalho de Costa (2014), após realizar uma pesquisa com 48 alunos de graduação em enfermagem, constatou que 94,59\% sinalizaram que a simulação contribuiu para a satisfação e para a segurança na prática de saúde, contra $2,70 \%$ que discordaram da afirmação, e 2,70\% que se mostraram indiferentes.

Com isso, para evitar danos e riscos aos pacientes, a utilização da tecnologia de ensino, sendo esta a simulação, oferece a 
oportunidade de inserção em um ambiente muito próximo do real, de forma controlada e segura (SANCHES, 2016). Atestando a ideia da autora citada, Runciman et al. (2009) acreditam que a simulação fundamenta-se em uma prática reflexiva, pois ao se mostrar paralela à realidade, acaba por desafiar o acadêmico a pensar de forma mais abrangente, buscando alternativas de resoluções.

Cabe ressaltar ainda que, de acordo com a estrutura da simulação, e a confiança, o rendimento e a competência que a mesma permite em casos clínicos reais, adicionados à necessidade de evitar erros na prática de saúde, a simulação pode acarretar melhoria da segurança do paciente (SOBRAL; CAMPOS, 2012).

Os achados desta pesquisa, bem como, o dos autores que guiaram a análise, demonstram os benefícios da simulação do processo de ensino e aprendizagem de enfermagem. Cabe aos docentes de enfermagem preparar a simulação de forma a tornála o mais próximo da realidade, desenvolvendo nos acadêmicos o pensamento crítico e o raciocínio clínico.

\section{Considerações finais}

A simulação no processo de ensino-aprendizagem na área da saúde abrange o desenvolvimento de técnicas e competências cognitivas capazes de transformar o ensino, tanto para o docente quanto para os acadêmicos e, por consequência, acarreta de forma direta na formação profissional dos enfermeiros.

As metodologias ativas com o uso da simulação na área da Enfermagem vêm aprimorando a perspectiva de construção do conhecimento, fazendo que professores e acadêmicos participem de forma efetiva, tornando o processo de ensino e aprendizagem mais flexível, crítico, reflexivo e versátil. Trata-se de uma estratégia inovadora com potencial transformador. Porém, mesmo exibindo uma metodologia tão benéfica, ainda há dificuldade de encontrar estudos que abordem o tema na área da saúde, mais precisamente na área da Enfermagem. 


\section{Referências}

AKHU-ZAHEYA, L. M., GHARAIBEH, M., \& ALOSTAZ, Z. M. Effectiveness of simulation on knowledge acquisition, knowledge retention, and self-efficacy of nursing students in Jordan. Clinical Simulation in Nursing, v. 9, n. 9, p. 335-342, 2013.

ARAÚJO, A. L. L. S.; QUILICI, A. P. O que é simulação e por que simular. In: . Simulação Clínica: do conceito à aplicabilidade. São Paulo: Editora Atheneu, p. 1-16, 2012.

ARCHANJO, D. R.; CORRÊA, C. L. As ciências neurológicas sob a pesquisa humanista: uma experiência pedagógica utilizando filmes. Fisioterapia e Pesquisa, São Paulo, v. 8, n. 2, p. 110-115, abr./jun. 2011.

BASS, J. Revolutionizing Engineering Science through Simulation. A Report of the National Science Foundation Blue ribbon Panel on Simulation-Based Engineering Science. Virginia, USA: National Science Foundation, 66p. 2006.

BELHOT, R. V.; FIGUEIREDO, R. S.; MALAVÉ, C. O. O uso da simulação no ensino de engenharia. In: Congresso Brasileiro de Ensino de Engenharia, XXIX Cobenge, p. 445-451, 2001.

BEZ, M. R. Construção de um Modelo para o Uso de Simuladores na Implementação de Métodos Ativos de Aprendizagem das Escolas de Medicina. Porto Alegre, 2013, 314s. Tese (Doutorado em Informática na Educação) - PGIE/ UFRGS, Porto Alegre, 2013.

BRADLEY, P. The history of simulation in medical education and possible future directions. Medical Education, v. 40, n. 3, p. 254-262, 2006.

BRANDT, B., LUTFIYYA M. N., KING J. A., CHIORESO C. A scoping review of interprofessional collaborative practice and education using the lens of Triple Aim. Journal of interprofessional care, n. 28, v. 5, p. 393-399, 2014.

Claro, C. M.; KROKOCZ, D. V. C.; TOFFOLETTO, M. C.; PADILHA, K. G. Eventos adversos em unidade de terapia intensiva: percepção dos enfermeiros sobre a cultura não punitiva. Revista da Escola de Enfermagem da USP, v. 45, n. 1, p. $167-72,2011$.

COSTA, R. R. O. A simulação realística como estratégia de ensinoaprendizagem em enfermagem (dissertação). Natal (RN): Universidade Federal do Rio Grande do Norte, 2014. 
COSTA, R. R. O.; MEDEIROS, S. M.; MARTINS, J. C. A.; MENEZES, R. M. P.; ARAÚJO, M. S. O uso da simulação no contexto da educação e formação em saúde e enfermagem: uma reflexão acadêmica. Revista Espaço para a Saúde, v. 16, n. 1, p. 59-65, 2015.

COUTINHO, V. R. D., MARTINS, J. C. A.; PEREIRA, M. D. F. C. R. Construção e Validação da Escala de Avaliação do Debriefing associado à Simulação (EADaS). Revista de Enfermagem Referência, v. IV, n. 2, p. 41-50, 2014.

COUTO, T. B. Simulação realística no ensino de emergências pediátricas na graduação. Dissertação (mestrado) - Faculdade de Medicina da Universidade de São Paulo, Programa de Pediatria, 76p, 2014.

DUDLEY, F. The Simulated Patient Handbook: A Comprehensive Guide for Facilitators and Simulated Patients. Radcliffe, 2012.

FErreira, C., CARVAlHO, J. M., CARVALHO, F. L. Q. Impacto da metodologia de simulação realística, enquanto tecnologia aplicada a educação nos cursos de saúde. II Seminário de tecnologias aplicadas em educação e saúde, 2015.

FERREIRA RP, GUEDES HM, OLIVEIRA DWD, et al. Simulação Realística como Estratégia de Ensino no Aprendizado de Estudantes da Área da Saúde. Revista de Enfermagem do Centro-Oeste Mineiro, v. 8, 2018.

GABA D. M. The future vision of simulation in healthcare. Quality and safety in health care, v. 13, n. 1, p. 2-10, 2004.

GOMES, C. O.; GERMANO, R. M. Processo ensino/aprendizagem no laboratório de enfermagem: visão de estudantes. Revista Gaúcha de Enfermagem, v. 28, n. 3, p. 401-408, 2007.

JONG, T.; JOOLINGEN, W. R. Scientific discovery learning with computer simulations of conceptual domains. Review of educational research, v. 68 , n. 2, p. 179-201, 1998.

LÉVY, P. O que é virtual? São Paulo: Editora 34, 2007.

ORTON E., MULHAUSEN P. E-learning virtual patients for geriatric education. Gerontology and geriatrics education, v. 28, n. 3, p. 73-88, 2008.

PADOVANI, O., CORRÊA, A. K. Currículo e Formação do Enfermeiro: Desafios das Universidades na Atualidade. Saúde e Transformação Social, v. 8, n. 2, 2017. 
PAIVA, M. R. F., PARENTE, J. R. F., BRANDÃO, I. R., \& QUEIROZ, A. H. B. Metodologias ativas de ensino-aprendizagem: revisão integrativa. Revista de Políticas Públicas, v. 15, n. 2, p. 145-153, 2016.

PEDUZZI M., CIAMPONE MHT. Trabalho em equipe e processo grupal. In: Kurcgant P., coordenadora. Gerenciamento em enfermagem, 2. ed. Rio de Janeiro: Guanabara Koogan, 2010, p. 105-20.

PEREIRA, L. A. P. Criação, desenvolvimento, aplicação e validação de um simulador computadorizado de realidade virtual para o ensino e treinamento de bloqueio do nervo alveolar inferior. Tese (doutorado), 103p - Universidade Estadual de Campinas, Faculdade de Odontologia de Piracicaba, 2016.

PRETO, L.; MAGALHÃES, C. P.; FERNANDES, A. A simulação de cuidados complexos: Uma nova ferramenta formativa. Sinais Vitais, Eiras (Coimbra), n. 89, p. 48-51, 2010.

QUILICI AP, ABRÃO K., TIMERMAN S., GUTIERREZ F. Simulação clínica: do conceito à aplicabilidade. São Paulo: Atheneu, 2012.

ROSSO, A.; TAGBLIEBER, J. Métodos Ativos e Atividades de Ensino. Perspectiva - Revista do Centro de Ciências e Educação, v. 10, n. 17, p. 37-46, 1992.

RUNCIMAN W., HIBBERT P., THOMSON R., VAN DER SCHAAF T., SHERMAN H., LEWALLE P. Towards an international classification for patient safety: key concepts and terms. Int J Qual Health Care, v. 21, p. 18-26, 2009.

SANCHES, M. O. Concepções do enfermeiro docente sobre o cuidado. Porto Alegre, 2006, 88s. Dissertação (Mestrado) - Escola de Enfermagem, Universidade Federal do Rio Grande do Sul, Porto Alegre, 2006.

SCALABRINI NETO, A. Simulação realística e habilidades na saúde, 1. ed. Rio de Janeiro, RJ: Atheneu, 2017.

SMITH SJ, ROEHRS CJ. High-fidelity simulation: factors correlated with nursing student satisfaction and self-confidence. Nursing education perspectives, v. 30 , n. 2, p. $76-78,2009$.

SOBRAL, F. R.; CAMPOS, C. J. G. Utilização de metodologia ativa no ensino e assistência de enfermagem na produção nacional: revisão integrativa. Revista da Escola de Enfermagem da USP, v. 46, n. 1, p. 208-218, 2012. 
SOUSA, J. E. R. B. Experiência de simulação clínica avançada em Enfermagem: satisfação e autoconfiança como resultados, 2015, 83s. Dissertação (Mestrado em Enfermagem) - Programa de Pós-Graduação em Enfermagem, Universidade Federal do Piauí, 2015.

STANFORD, P. G. Simulation in nursing education: A review of the research. The Qualitative Report, v. 15, n. 4, p. 1.006-1.011, 2010.

TERRA, M. G. et al. O significado de cuidar no contexto do pensamento complexo: novas possibilidades para a enfermagem. Texto e Contexto Enfermagem, v. 15, n. esp., p. 164-169, 2006.

VERSIANI, A.; FACHIN, R. C. Avaliando aprendizagem em simulações empresariais. Cadernos EBAPE - BR, v. 5, n. spe., p. 1-13, 2007.

ZIV, A.; DAVID, B.; ZIV, M. Simulation Based Medical Education: an opportunity to learn from errors. Medical Teacher, v. 27, n. 3, p. 193-199, 2005.

Submetido em: 23-10-2018

Aceito em: 30-11-2018 